\title{
Effectiveness of two new types of sealants: retention after 2 years
}

\author{
Xi Chen • Minquan Du • Mingwen Fan • Jan Mulder • \\ Marie-Charlotte Huysmans • Jo E. Frencken
}

Received: 28 February 2011 / Accepted: 20 October 2011 /Published online: 29 November 2011

(C) The Author(s) 2011. This article is published with open access at Springerlink.com

\begin{abstract}
The hypotheses tested were: survival rate of fully and partially retained glass-carbomer sealants is higher than those of high-viscosity glass-ionomer, with and without energy supplied, and that of resin composite; survival rate of fully and partially retained sealants of high-viscosity glass-ionomer with energy supplied is higher than those without energy supplied. The randomized clinical trial covered 407 children, with a mean age of 8 years. The evaluation took place after $0.5,1$ and 2 years. Survival of sealant material in occlusal and in smooth surfaces, using the traditional categorization (fully and partially retained versus completely lost sealants) and the modified categorization (fully and more than $2 / 3$ of the sealant retained versus completely lost sealants), were dependent variables. The Kaplan-Meier survival method was used. According to
\end{abstract}

Electronic supplementary material The online version of this article (doi:10.1007/s00784-011-0633-9) contains supplementary material, which is available to authorized users.

X. Chen $\cdot$ M. Du $\cdot$ M. Fan $(\bowtie)$

Preventive Department, Key Laboratory for Oral Biomedical

Engineering, School and Hospital of Stomatology,

Wuhan University,

Luoyu Road 237,

430079 Wuhan, Hubei, People's Republic of China

e-mail: kqyywjtx@hotmail.com

J. Mulder $\cdot$ J. E. Frencken $(\bowtie)$

Department of Global Oral Health, College of Dental Sciences,

Radboud University Nijmegen Medical Centre,

P.O. Box 9101, 6500 HB Nijmegen, The Netherlands

e-mail: j.frencken@dent.umcn.nl

M.-C. Huysmans

Department of Restorative Dentistry and Endodontology,

College of Dental Sciences,

Radboud University Nijmegen Medical Centre,

Nijmegen, The Netherlands both categorizations of partially retained sealants, the survival of completely and partially retained resin composite sealants in occlusal and in smooth tooth surfaces was statistically significantly higher, and those of glasscarbomer sealants lower, than those of sealants of the other three groups. There was no statistically significant difference in the survival rates of completely and partially retained high-viscosity glass-ionomer sealants with and without energy supplied in occlusal and in smooth surfaces. After 2 years, glass-carbomer sealant retention was the poorest, adding energy to high-viscosity glass-ionomer sealant did not increase the retention rate and resin composite sealants were retained the longest. We suggest the use of the modified categorization of partially retained sealants in future studies. It seems not necessary to cure high-viscosity glass-ionomer sealants. The use of glasscarbomer sealants cannot be recommended yet.

Keywords Atraumatic restorative treatment - Resin composite sealant . Glass-ionomer sealant . Glass-carbomer sealant . Caries prevention . Sealant retention

\section{Introduction}

It is widely accepted that sealing pits and fissures in newly erupted molars is effective in preventing carious lesion development [1] and progression [2, 3]. The two most commonly used materials for sealing pits and fissures are based on resin and glass-ionomer. The retention of resinbased sealant material is generally considered to be higher than those of low- and medium-viscosity glass-ionomerbased materials.

Further reduction of the development of carious lesions in pits and fissures requires the use of sealant materials that 
adhere longer to the enamel. One such modification has been the introduction of high-viscosity glass-ionomer. The powder-to-liquid ratio of this type of material has been elevated, in order to produce a high-viscosity glass-ionomer with improved physical-mechanical performances, including prolonged retention to enamel [4]. Marketed over 15 years, it has proved to be fully and partially retained by up to $72 \%$ after 3 years [5].

Initial studies have shown that a high-viscosity glassionomer sets faster if heat is applied during the setting procedure [6]. Using a high energy light to cure glassionomer increases the temperature in the cement, which may also shorten the setting time and improve the adhesion of the glass-ionomer to the enamel. Another reported event regarding glass ionomers relates to changes in the composition of glass-ionomer sealants over time, observed in vivo and identified when using SEM, as 'enamel-like structures' [7]. This led to the development of another new glassionomer-based material called glass-carbomer. Fluorapatite has been added to its nano-sized reduced powder particles, prompting the manufacturer to claim that the clinical performance of the material is excellent.

These two types of sealants have been tested in vitro and showed low marginal leakage at the enamel-sealant interface for high-viscosity glass-ionomer with energy added and a non-interpretable outcome for glasscarbomer [8]. However, the effects of the addition of energy to this relatively new high-viscosity glass-ionomer material and that of glass-carbomer have not previously been tested for sealing pits and fissures in vivo. As the Chinese government launched a nationwide school sealant programme in 2010 and as a recall system for monitoring the effectiveness of sealants in schools is rarely available, the use of a material with a long retention time was desirable, for ensuring the sealant's preventive effect over a long period.

The hypotheses tested were: (1) the cumulative survival rate of fully and partially retained glass-carbomer sealants is higher than those of high-viscosity glass-ionomer sealants, with and without energy supplied, and that of resin composite after 2 years; (2) the cumulative survival rate of fully and partially retained high-viscosity glass-ionomer sealants with energy supplied is higher than that of highviscosity glass-ionomer sealants without energy supplied, after 2 years.

\section{Materials and methods}

Study population and treatment allocation Wuhan, the capital of Hubei Province, China, has a population of about 8 million people. The average GDP of its citizens increased from US \$89 in 1978 to US \$2,777 in 2009.
This study was conducted in five public schools in the urban area of Wuhan.

The sample of subjects for the present investigation resulted from data derived from an oral health epidemiological survey of grade 2 children attending these schools. The sampling procedure was based on the caries experience of the children, assessed according to the Atraumatic Restorative Treatment (ART) caries criteria (Table 1), and on the morphology of pits and fissures in the first permanent molars, assessed according to the criteria described by Symons et al. [9]. The inclusion criteria for enrolment in the treatment study were: a fully erupted first permanent molar, no dentine caries lesion in pits and fissures of these molars, deep and/or intermediate pits or fissures and a $\mathrm{dmft} \geq 2$. The latter two criteria indicate a state of medium to high caries risk. For a child to be eligible for inclusion in the study, all these criteria needed to be present. Children's parents or guardians received and signed individual informed consent forms containing information about the aim of the study and the treatment procedures. Children whose parents declined to sign the consent form were excluded from the study.

The study was a randomized clinical trial with sealants clustered in each child. Per school, the first author randomly allocated each included child to one of the four sealant groups, using a list obtained after block randomisation (12 children per block for three operators) and prepared by a statistician who did not do the analyses. The list was contained in an envelope and opened on the first day of the trial. Per child, all first permanent molars with

Table 1 Dental caries diagnostic index used in the present study (ART caries assessment criteria)

\begin{tabular}{|c|c|c|}
\hline \multicolumn{2}{|l|}{ Code } & \multirow[t]{2}{*}{ Description } \\
\hline Permanent & Deciduous & \\
\hline 0 & A & Sound surface \\
\hline 1 & B & $\begin{array}{l}\text { Early enamel lesion. White/opaque or } \\
\text { brownish/dark lesion in enamel only, } \\
\text { including loss of tooth surface; considered } \\
\text { being active or inactive }\end{array}$ \\
\hline 2 & $\mathrm{C}$ & $\begin{array}{l}\text { Carious lesion involving the dentine slightly; } \\
\text { lesion cannot be penetrated with CPI probe }\end{array}$ \\
\hline 3 & $\mathrm{D}$ & $\begin{array}{l}\text { Dentinal lesion; lesion can be penetrated with } \\
\text { CPI probe }\end{array}$ \\
\hline 4 & $\mathrm{E}$ & $\begin{array}{l}\text { Dentinal lesion: pulp possibly or definitely } \\
\text { exposed. }\end{array}$ \\
\hline 5 & $\mathrm{~F}$ & Restoration \\
\hline 6 & G & Sealant \\
\hline 7 & $\mathrm{H}$ & Missing due to caries \\
\hline 8 & 8 & Unerupted permanent tooth \\
\hline 9 & 9 & Unable to make diagnosis \\
\hline
\end{tabular}


eligible pits and fissures were sealed according to one procedure only. The study was approved by the Research Ethics Committee of Wuhan University, Reference No. 200704, and was registered at the Dutch Trial Registration Centre, with reference no. 1411.

As the application procedure of placing glass-ionomer and glass-carbomer sealants was new to the three operating dentists and the two dental ancillaries, a 4-week laboratory and field training programme in a primary school, which further included the process of recording data, was carried out before the start of the clinical trial. Portable equipment, including operating light and adjustable bed, was used in placing the sealants in the first permanent molars, on the school premises, within a period of 2 months. Sealants were placed in pits and fissures of occlusal surfaces and in those of buccal and palatal fissures (smooth surfaces). The number of sealants placed is presented in Table 2, according to the operator, school and type of jaw. Children received instructions about good oral health behaviour, and final year dental students showed them, on an individual basis, how to clean their teeth. Children were requested to go to the Dental Hospital for receiving restorative care after sealants had been placed and for treatment of the failed sealants (dentine caries lesion development) after each evaluation time.

\section{Sealant procedures}

Group 1-glass-ionomer: Ketac Molar Easymix ${ }^{\circledR}$ (3MESPE, Seefeld, Germany) This is a test group. Sealant application followed the ART sealant procedure [10]. The occlusal surface and pits and fissures were cleaned with wet cotton wool pellets and a no. 6 explorer, dried with dry cotton wool pellets, conditioned with a moist pellet dipped in the glass-ionomer liquid for $10 \mathrm{~s}$, then washed twice with wet cotton wool pellets and dried with dry ones. Glassionomer powder and liquid were mixed within $30 \mathrm{~s}$, applied to the surface with an applier/carver ART instrument (Henry Schein, Chicago, IL, USA) and firmly pressed into place for 5-10 s by a petroleum jelly coated index finger (press-finger technique). Excess material and the petroleum jelly coated top layer were removed, using the applier/ carver ART instrument. The surface was burnished, using the smooth curved angle of the ART applier instrument and finally, covered with a new layer of petroleum jelly. Children were advised not to eat or bite for at least $1 \mathrm{~h}$.

Group 2-glass-ionomer plus added energy: Ketac Molar Easymix ${ }^{\circledR}$ plus LED high energy curing light, Elipar ${ }^{\mathrm{TM}}$ Freelight 2, (3MESPE, Seefeld, Germany), producing $850 \mathrm{~mW} / \mathrm{cm}^{2}$ This is a test group. The sealant application described for group 1 was followed, except that the surface was cured for $60 \mathrm{~s}$ after burnishing and before placement of the layer of petroleum jelly. The wave strength of the LED curing light was checked every week to ensure that the wave strength was above $750 \mathrm{~mW} / \mathrm{cm}^{2}$.

Group 3-glass-carbomer: Glass Carbomer ${ }^{\circledR}$ (First Scientific Dental, Elmshorn, Germany) This is a test group. Cleaning of the occlusal surface and pits and fissures was done as described for group 1, using cotton wool rolls for isolation. Thereafter, a cotton pellet dipped in Glass Carbomer Tooth Cleaner (First Scientific Dental, Elmshorn, Germany) was wiped over the tooth surface for $20 \mathrm{~s}$ for further cleaning. Washing and drying of the surface with two wet and dry cotton pellets, respectively, followed. The Glass Carbomer ${ }^{\circledR}$ capsule was activated, mixed for $15 \mathrm{~s}$ in a RotomixTM (3MESPE, Seefeld, Germany), extruded within $1 \mathrm{~min}$ from the start of mixing, onto the tooth surface, spread into a thin film, covered with Glass Garbomer Surface Gloss (First Scientific Dental, Elmshorn, Germany) and held under finger pressure for 5-10 s. The applier/carver ART instrument was used to remove excess material, and its smooth curved angle, for burnishing the surface. Thereafter, the material was light cured for $75 \mathrm{~s}$, using the same LED light as used in group 2, and the same advice was given as group 1 received.

Group 4-resin composite, Clinpro ${ }^{\circledR}$ (3MESPE, St. Pauls, Miniapolis, MN, USA) This is the control group. The occlusal surface and pits and fissures were cleaned with a rotating brush Prophy Angle (3MESPE, Wuhan, China) and a no. 6 explorer using a suction device for isolation. After thorough rinsing and drying, the occlusal surface was acid etched, using Scotchbond ${ }^{\mathrm{TM}}$ etchant (3MESPE, St. Pauls, Miniapolis, MN, USA) for $20 \mathrm{~s}$, rinsed and dried. The sealant material was placed in the pits and fissures, manipulated with an explorer to free potential air bubbles
Table 2 The number of sealants placed by operator, school and type of jaw at baseline

\begin{tabular}{|c|c|c|c|c|c|c|c|c|c|c|}
\hline & \multicolumn{3}{|c|}{ Operator } & \multicolumn{5}{|c|}{ School } & \multicolumn{2}{|l|}{ Jaw } \\
\hline & 1 & 2 & 3 & 1 & 2 & 3 & 4 & 5 & upper & lower \\
\hline Noc & 437 & 455 & 452 & 335 & 285 & 238 & 188 & 298 & 674 & 670 \\
\hline Nsm & 228 & 115 & 172 & 146 & 120 & 93 & 52 & 104 & 159 & 356 \\
\hline Total & 665 & 570 & 624 & 481 & 405 & 331 & 240 & 402 & 833 & 1,026 \\
\hline
\end{tabular}


and cured for $20 \mathrm{~s}$ with the LED curing light $1 \mathrm{~mm}$ above the surface. Bite adjustment was carried out, using carbon paper and rotary instruments.

Evaluation The coverage of the pits and fissures with the sealant material was recorded at baseline. The sealant retention criteria used are presented in Table 3 and were applied to each of the three sections (mesial-central-distal) into which the occlusal surface was arbitrarily divided. Teeth were cleaned with a toothbrush and toothpaste before the start of the evaluation. Remaining visible debris and plaque were removed with the aid of an explorer. Before examination, the sealed tooth surface was dried, using a piece of cotton tightly attached to the end of a stick. If sealant material was judged to have disappeared completely from the tooth surface or from a section of it, the re-exposed pits and fissure(s) were dried with an air syringe and judged again. The examination site was well illuminated by an intraoral light attached to a mirror handle (Kudos, Hong Kong).

The evaluation was done after $0.5,1$ and 2 years by the same calibrated and experienced independent evaluators, one from Hong Kong and one from Beijing Dental School, who were kept uninformed about the sealant materials used. They were assisted by trained recorders who had also assisted the operators. Before and during the morning of the first day of evaluation years 1 and 2, evaluators were recalibrated by examining the first arriving children twice. In the presence of the senior investigator, differences were discussed and consensus was reached when complete agreement covering a series of $4-5$ children was shown.

The intra-evaluator consistency in assessing sealant retention was assessed by re-examining 199 and 190 pairs of sealed tooth sections for evaluators 1 and 2 respectively, while the inter-evaluator consistency was assessed by reexamining 1,231 pairs of sealed tooth sections. The kappa coefficient for intra-evaluator consistency was 0.89 and 0.93 , and 0.62 for the inter-evaluator consistency. Figure 1 shows a flowchart of the number of participants and tooth surfaces sealed for the four treatment groups and number of dropouts at each evaluation stage of the study.

Statistical analysis A power calculation preceded the sampling procedure. On the basis of an accepted difference of $15 \%$ between the survival of caries lesion-free pits and fissures sealed with high-viscosity glass-ionomer (94\%) and composite resin (79\%) after 5 years [10], a total of 77 sealants per group were needed. Glass-carbomer and high-viscosity glass-ionomer with light-curing were new, and sealants placed according to these procedures were expected to cause a higher survival of carious lesion-free pits and fissures $(99 \%)$ than that of high-viscosity glass-ionomer without light-curing (94\%) after 5 years. Consequently, 208 sealants per group were required. Using the survival percentages and a power of $80 \%$, tested in two ways, a dropout of $30 \%$ after 5 years and a correction for the use of the dependency of measurements of $20 \%$, it was found that the maximum number of sealants per group would be 312. Moreover, depending on the number of eligible sealants per child, the number of children per group would be 156 (two sealants) or 104 (three sealants).

The data were entered into a database, checked for errors and analysed by a statistician, who was blinded to the sealant procedure, using SAS software (version 9.2). Statistical analysis, aimed at describing the survival curves of the four types of sealants, was carried out twice for sealants on occlusal surfaces only. Analysis was done once for the traditional categorization of completely retained (retention code 1 for all three sections) and partially retained (combinations of retention codes containing 1, 2 or 3 for the three sections) sealants, and those completely lost (retention codes 4,5 or 6 for all three sections). It was also done once according to a modification: 'completely retained sealants' was assessed as in the traditional categorization but the partially retained sealants (retention codes having a code 1 for two sections and a code 2 or 3 for the remaining section) and those completely lost (retention
Table 3 Evaluation criteria for assessing sealant retention

\begin{tabular}{ll}
\hline Score & Criteria \\
\hline \multicolumn{2}{l}{ Tooth need to be cleaned and dried with a piece of cotton } \\
1 & Pits and fissures completely covered with material \\
2 & Pits and fissures partly visible. Sharp fracture edge (creating plaque retention site) \\
3 & Pits and fissures partly visible. Crumbled fracture edge (not creating plaque retention site) \\
4 & Pits and fissures totally visible \\
If score & has been given then pits and fissures are re-observed using compressed air \\
5 & Pits and fissures totally covered with remnants \\
6 & Pits and fissures partly covered with remnants \\
7 & Other treatment performed \\
9 & Unable to diagnosis \\
\hline
\end{tabular}




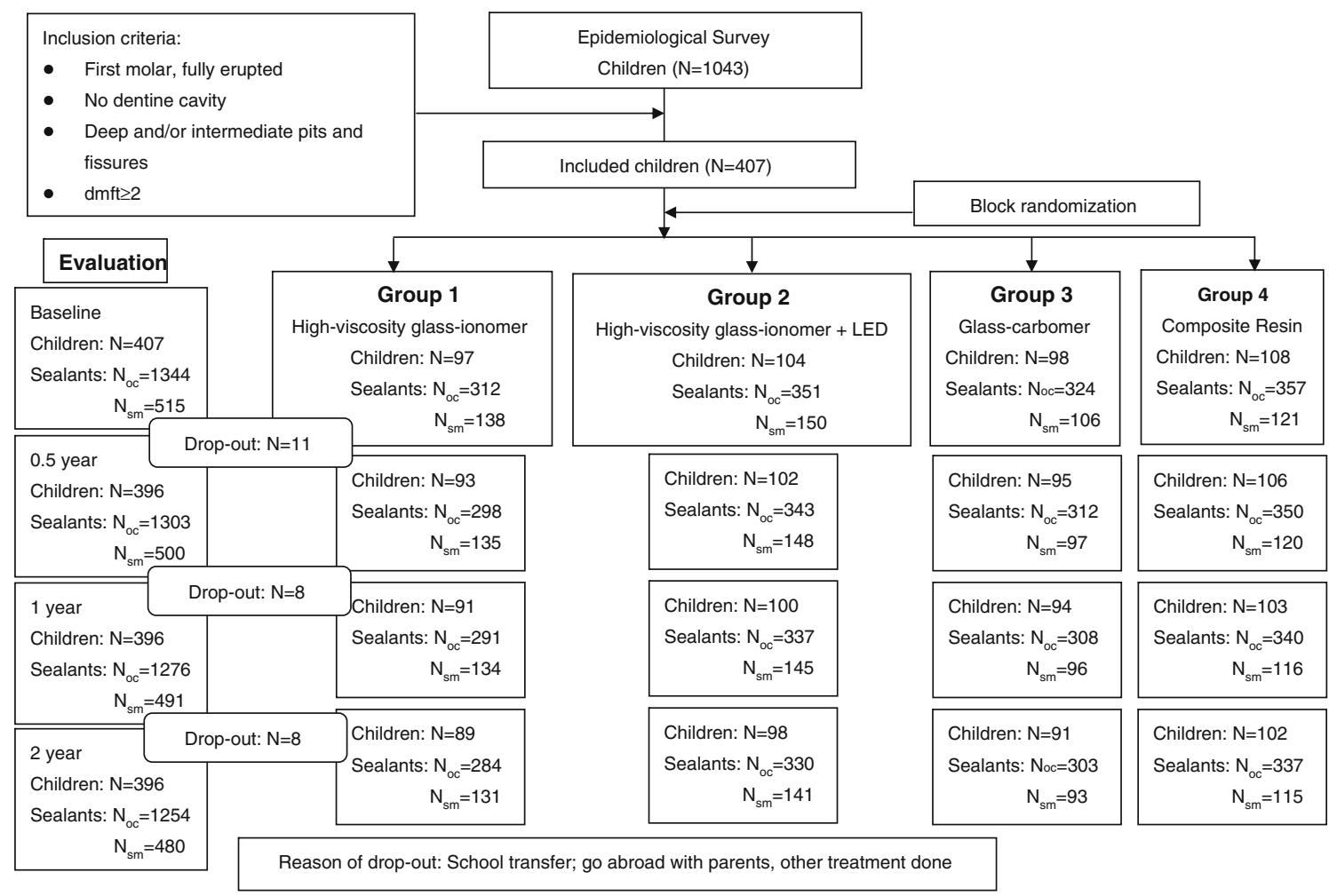

Fig. 1 Consort flow diagram of current investigation. ( $N$ number; $N_{o c}$ number of sealants in occlusal surface; $N_{s m}$ number of sealants in smooth surface)

codes having at least one code 4, 5 or 6 in one of the three sections) were assessed differently.

The data were censored, owing to dropouts and the restricted general follow-up period of 2 years. Longitudinal data series can be interrupted. Some series can be interpreted; others cannot. In the present study $21 / 15,807$ longitudinal series of sealant retention sections could not be interpreted. The 21 missing data were randomly allocated to one of the available scores of the longitudinal series through the flipping of a coin. The Kaplan-Meier survival method was applied in estimating survival percentages. Owing to the dependency of data on different sealants in one child, the Greenwood approximation for the standard error (SE) of the survival percentages was not valid [11]. Therefore, the jackknife method [12] (leaving one child out) was applied in calculating the SEs.

Gender, school (1-5), type of jaw (maxilla/mandible), sealant procedure (1-4) and operator (1-3) were the independent variables, while survival of sealant material in occlusal and in smooth surfaces, according to the traditional categorization, and the survival of sealant material in occlusal surfaces, in accordance with the modified categorization, were the dependent variables. The univariate chi-squares for the Wilcoxon test for censored data were used to test the effect of the independent variables on the survival of the sealant material. The $t$ test was used to test for the difference between the sealant material survival percentages of the four sealant procedures.

\section{Results}

Disposition of subjects A total of 407 children with an average age of 8.0 years (range, 7.0-9.1 years) participated in the trial. In total, 1,352 first permanent molars were sealed (mean $=3.3$ first permanent molars per child). The percentages of children with 1,2,3 and 4 first permanent molars sealed at baseline were 5.2, 16.0, 20.4 and 58.5, respectively. The dropout percentages of children and sealants over the two study years are presented in Fig. 1 and were regarded as being low $(6.6 \%$ of children and $6.8 \%$ of sealants at evaluation year 2). No difference in caries experience was observed between the four groups at baseline $\left(\mathrm{d}_{2} \mathrm{mft}: p=0.95 ; \mathrm{D}_{2}\right.$ MFT: $p=0.68 ; \mathrm{d}_{3} \mathrm{mft}: p=0.86$; $\mathrm{D}_{3}$ MFT: $\left.p=0.47\right)$. The mean $\mathrm{d}_{2} \mathrm{mft}$ score ranged from 6.2 to 6.4; that of $\mathrm{d}_{3} \mathrm{mft}$ from 4.7 to 5.0 ; that of $\mathrm{D}_{2} \mathrm{MFT}$ from 0.7 to 0.9 and that of $\mathrm{D}_{3} \mathrm{MFT}$ was either 0.1 or 0.2 .

Cumulative survival of completely and partially retained sealants according to the traditional categorization Table 4 shows the cumulative survival percentages and SEs, calculat- 
ed using the jackknife procedure, of sealants completely and partially retained in pits and fissures in occlusal and smooth surfaces of the first permanent molars over a period of 2 years, in accordance with the traditional categorization of completely and partially retained sealants, for the four treatment groups.

The analyses of the survival of sealant material in occlusal surfaces on the independent variables showed a treatment group effect $(p<0.0001)$, an operator $(p<0.0001)$ and a school effect $(p<0.0001)$ and no gender $(p=0.66)$ or type of jaw $(p=0.14)$ effect. The cumulative survival of completely and partially retained resin composite sealants in occlusal and in smooth tooth surfaces after 2 years was statistically significantly higher than those of the other three groups of sealants (Table 4). The cumulative survival of completely and partially retained glass-carbomer sealants in occlusal and in smooth tooth surfaces was statistically significantly lower than those of sealants of the other three groups (Table 4). After 2 years there was no statistically significant difference in the survival rates of completely and partially retained high-viscosity glass-ionomer sealants with and without energy supplied, in occlusal $(p=0.23)$ and in smooth surfaces $(p=0.37)$. The cumulative survival of completely and partially retained sealants in both occlusal and smooth surfaces was statistically significantly higher for operator 1 than for the other two operators $(p \leq 0.006)$, as it was in four schools compared to one school (school 2) $(p=0.001)$.

Cumulative survival of completely retained sealants according to the modified categorization In accordance with the modified categorization of completely and partially retained sealants for the four treatment groups, Table 5 shows the cumulative survival percentages and SEs, calculated according to the jackknife procedure, of sealants completely and partially retained in pits and fissures in occlusal surfaces of the first permanent molars over a period of 2 years. As was expected, retention rates were lower overall. However, the statistical analyses showed essentially similar results to those of the traditional analysis, with the same significant effects. Only the operator effect was different, with the cumulative survival of sealants in occlusal surfaces being significantly lower for operator 2 than for the other two operators $(p \leq 0.009)$. The retention rates of completely and partially retained sealants assessed according to the traditional categorization were higher than those assessed according to the modified categorization, in all four treatment groups.

\section{Discussion}

Methodology In sealant retention studies over time, analyses usually identify completely and partially retained 
Table 5 The cumulative survival percentages and SEs, calculated using the jackknife procedure, of sealants that have been completely and partially retained in pits and fissures of occlusal surfaces in the first permanent molars over a period of 2 years using the modified categorization of completely and partially retained sealants

\begin{tabular}{lllll}
\hline $\begin{array}{l}\text { Group } \\
\text { Year }\end{array}$ & $\begin{array}{l}1 \\
\text { High-viscosity glass-ionomer } \\
\begin{array}{l}\text { Occlusal } \\
\% \pm \mathrm{SE}\end{array}\end{array}$ & $\begin{array}{l}2 \\
\text { High-viscosity glass-ionomer + LED } \\
\text { Occlusal } \\
\% \pm \mathrm{SE}\end{array}$ & $\begin{array}{l}3 \\
\text { Glass-carbomer } \\
\text { Occlusal } \\
\% \pm \mathrm{SE}\end{array}$ & $\begin{array}{l}4 \\
\text { Composite resin } \\
\text { Occlusal } \\
\% \pm \mathrm{SE}\end{array}$ \\
\hline $0-0.5$ & $81.2 \pm 2.6$ & $84.0 \pm 2.4$ & $38.8 \pm 4.0$ & $86.3 \pm 2.2$ \\
$0.5-1$ & $66.1 \pm 3.2$ & $69.1 \pm 2.9$ & $17.9 \pm 2.8$ & $76.6 \pm 2.7$ \\
$1-2$ & $44.6 \pm 3.4^{\mathrm{bd}}$ & $45.0 \pm 3.2^{\mathrm{bd}}$ & $8.4 \pm 1.9^{\mathrm{bc}}$ & $55.7 \pm 3.1^{\text {ad }}$ \\
\hline
\end{tabular}

$S E$ standard errors

$p_{\mathrm{a}-\mathrm{b}} \leq 0.004 ; p_{\mathrm{c}-\mathrm{d}}<0.001$

sealants as those having survived and sealants that have completely disappeared as those which have failed. The group of partially retained sealants range from those in which pits and fissures are almost completely covered with sealant material to those in which pits and fissures are almost completely re-exposed to the oral environment. The analysis covering this wide range in states of partially retained sealants makes the determination of sealants that are considered to have survived unrealistic. It is obvious that in pits and fissures almost completely covered with a sealant, the chance for carious lesion development is higher than in those from which the sealant material has almost completely disappeared. We therefore decided to redetermine the effectiveness of partially retained sealants. This was possible because we had divided the occlusal surface into three equal sections, which led to three retention scores per sealant. Based on the assumption that the absence of sealant material from one section would seriously increase the chance for caries lesion development in that surface, the cutoff point for partially retained sealant included two or more sections completely covered with sealant materials. The analyses based on this latter dichotomy showed lower survival rates than obtained for the traditional dichotomy of sealants completely disappeared versus those that have at least some material left. We think that the modification made to the description of partially retained sealants does more justice to the performance of a sealant and we therefore recommend its use in future studies covering sealant retention.

The study design was a clustered randomized clinical trial, with the sealants in the oral cavity as the cluster. In such a situation, sealants are not independent units and compensatory measures should be taken in the statistical analysis. The jackknife procedure is a proven way of dealing with the dependency of the clustered data which results in an increase in the value of the standard error.

The dropout rate after 2 years was very low compared with those of other clinical sealant retention studies, which adds to the reliability of the outcomes. Another factor promoting the reliability of the outcomes is the high kappa coefficient value for intra- and inter-evaluator consistency. Blinding of the evaluators was accomplished by not informing them about the sealant materials used and by printing incorrect information regarding the materials on the record form. The operators were not blinded but blinding of the statistician was achieved, as coded numericals were provided and were decoded only at the end of the analyses. It is thus fair to conclude that the internal and external validity of the outcomes is substantial.

Outcomes The first hypothesis was rejected. Glasscarbomer sealants were retained shorter than resin composite sealants and ART high-viscosity glass-ionomer sealants, with and without energy supplied, after 2 years. The second hypothesis was rejected. ART Sealants of high-viscosity glass-ionomer with energy supplied did not survive longer than ART sealants of high-viscosity glass-ionomer without energy supplied, after 2 years. Other remarkable findings were the good performance of resin-based sealants and the higher survival in occlusal than in smooth surfaces, of retained sealants of all types after 2 years. In the in vitro study that preceded the present in vivo study, marginal leakage at the enamel-glass-carbomer sealant interface was non-interpretable [8]. The body of the glass-carbomer sealants contained types of fracture lines. These were, however, not filled with silver nitrate, used as the tracer, but appeared to be glassy. Discussion with the originator of the glass-carbomer revealed that the batches sent to us were produced at a below-standard level, which may account for the low survival rates observed in the present study. The glassy lines observed in the glass-carbomer sealants in the in vitro study were due to resin infiltration into the surface glass. This disrupted the setting and adhesion reactions of the material. It explains why glass-carbomer in this study stayed rather soft for a long time after the required curing time was over. This experience points to the responsibility of companies to maintain a very high standard of quality control of their products at all times and that they should destroy materials of inferior quality. 
We have no explanation as to why the retention of all types of sealants was higher in occlusal than in smooth surfaces. For resin composite sealants, this finding is in agreement with the results reported for retained sealants in buccal surface pits and fissures, with difficulties in moisture control being cited as a possible reason [13].

The $86 \%$ retention rate of resin composite sealants after 2 years appears to be in line with results reported in the Cochrane review (71-85\%) [1]. The 78\% retention rate, after 2 years, of high-viscosity glass-ionomer sealants applied according to the ART approach is in line with that of $82 \%$ reported in the meta-analyses of ART high-viscosity glassionomer sealants [5]. As retention is a proxy outcome for the desired outcome of caries prevention, until the results of the analyses on the incidence of caries lesions become available, determining which of the sealant procedures will be most effective for use in China will not be possible.

Although ample time was devoted to training the three operators well in all four sealant procedures, an operator effect was observed. Such an effect is unwanted but seems difficult to avoid, considering reported operator effects regarding resin composite sealants [14] and glass-ionomer sealants [15]. Perhaps an extended training session longer than that carried out in the preparation stage of the present study would have eliminated the operator effect. It is unclear why the survival of fully and partially retained sealants was lower in school 2 than in the other schools. School 2 was the first school visited and maybe there was still a learning effect, although an interaction between operators and schools was not present.

Conclusion We conclude that, after 2 years, sealants produced with resin composite were retained for the longest period, retention of those produced with glass-carbomer was the poorest and adding energy to high-viscosity glass-ionomer ART sealant does not increase the retention rate. We favour the use of the modified categorization of partially retained sealants in analysing and reporting of clinical sealants studies. More studies on this topic are, however, required.

\begin{abstract}
Acknowledgements We thank Dr. Ye Lu, Dr. Li Jiqi and Dr. Hu Xuan, the dental assistants and final year dental students, for their pleasant and valuable contribution. We have appreciated the donation of dental materials from 3MESPE, China and Glass-Carbomer N.V., the Netherlands. The study was financed by the grant from the Ministry of Science and Technology, China (2007BA128B00) and from the Netherlands Academy of Science (08CDP011) and from the Radboud University Nijmegen (RL000045), the Netherlands.
\end{abstract}

Declaration of interests Authors declare that they have no conflict of interest.
Open Access This article is distributed under the terms of the Creative Commons Attribution Noncommercial License which permits any noncommercial use, distribution, and reproduction in any medium, provided the original author(s) and source are credited.

\section{References}

1. Ahovuo-Saloranta A, Hiiri A, Nordblad A, Mäkelä M, Worthington $H$ (2008) Pit and fissure sealants for preventing dental decay in the permanent teeth of children and adolescents. Cochrane Database Syst Rev 4:CD001830

2. Griffin SO, Oong E, Kohn W, Vidakovic B, Gooch BF, Bader J, Clarkson J, Fontana MR, Meyer DM, Rozier RG, Weintraub JA, Zero DT (2008) The effectiveness of sealants in managing caries lesions. J Dent Res 87:169-174

3. Oong EM, Griffin SO, Kohn WG, Gooch BF, Caufield PW (2008) The effect of dental sealants on bacteria levels in caries lesions: a review of the evidence. J Am Dent Assoc 139:271-278

4. Peez R, Frank S (2006) The physical-mechanical performance of the new Ketac Molar Easymix compared to commercially available glass ionomer restoratives. J Dent 34:582-587

5. van't Hof MA, Frencken JE, van Palenstein WH, Holmgren CJ (2006) The ART approach for managing dental caries: a metaanalysis. Int Dent J 56:345-351

6. Algera TJ, Kleverlaan CJ, de Gee AJ, Prahl-Andersen B, Feilzer AJ (2005) The influence of accelerating the setting rate by ultrasound or heat on the bond strength of glass ionomers used as orthodontic bracket cements. Eur J Orthod 27:472-476

7. van Duinen RN, Davidson CL, De Gee AJ, Feilzer AJ (2004) In situ transformation of glass-ionomer into an enamel-like material. Am J Dent 17:223-227

8. Chen X, Cuijpers V, Fan M, Frencken JE (2010) Marginal leakage of two newer glass-ionomer-based sealant materials assessed using micro-CT. J Dent 38:731-735

9. Symons AL, Chu CY, Meyers IA (1996) The effect of fissure morphology and pretreatment of the enamel surface on penetration and adhesion of fissure sealants. J Oral Rehabil 23:791-798

10. Beiruti N, Frencken JE, van't Hof MA, Taifour D, van Palenstein Helderman WH (2006) Caries-preventive effect of a one-time application of composite resin and glass ionomer sealants after 5 years. Caries Res 40:52-59

11. Greenwood M (1926) The errors of sampling the survivorship tables [appendix 1] Reports on Public Health and Medical Subjects, 33. Her Majesty's Stationery Office, London

12. Efron B (1982) The jackknife, the bootstrap and other resampling plans. SIAM-NSF, Philadelphia

13. Wendt LK, Koch G, Birkhed D (2001) On the retention and effectiveness of fissure sealants in permanent molars after 1520 years: a cohort study. Community Dent Oral Epidemiol 29:302-307

14. Folke BD, Walton JL, Feigal RJ (2004) Occlusal sealant success over 10 years in a private practice: comparing longevity of sealants placed by dentists, hygienists, and assistants. Pediatr Dent 26:426-432

15. Frencken JE, Makoni F, Sithole WD, Hackenitz E (1998) Threeyear survival of one-surface ART restorations and glass-ionomer sealants in a school oral health programme in Zimbabwe. Caries Res 32:119-126 